\title{
Taking in the trash: bioreactors for the mass production of clinical-grade extracellular vesicles
}

\author{
Matthew J. Robeson, Michael E. Davis \\ Wallace H. Coulter Department of Biomedical Engineering, Emory University and Georgia Institute of Technology, Atlanta, GA \\ 30322 , USA.
}

Correspondence to: Prof. Michael E. Davis, Wallace H. Coulter Department of Biomedical Engineering, Emory University and Georgia Institute of Technology, 2015 Uppergate Drive, Atlanta, GA 30322, USA. E-mail: medavis@emory.edu

How to cite this article: Robeson MJ, Davis ME. Taking in the trash: bioreactors for the mass production of clinical-grade extracellular vesicles. J Cardiovasc Aging 2022;2:11. https://dx.doi.org/10.20517/jca.2021.32

Received: 29 Nov 2021 Accepted: 1 Dec 2021 Published: 7 Jan 2022

Academic Editor: Ali J. Marian Copy Editor: Yue-Yue Zhang Production Editor: Yue-Yue Zhang

In recent years, the quest for the next generation of therapeutics for cardiovascular diseases has focused heavily on extracellular vesicles (EVs), and for good reason. Formed by the inward budding of the endosomal membrane, EVs are small secreted vesicles about 50-150 nm in diameter, containing a myriad of bioactive cargo, including lipids, proteins, and micro-RNAs (miRs) ${ }^{[1]}$. Though their existence has been known since at least the early 1980s, EVs were originally considered little more than "garbage bins", or a means by which cells package and expel unnecessary proteins and other molecules ${ }^{[2]}$. In the intervening years, an increasing body of evidence has identified EVs as a primary mediator of paracrine signaling between cells. Secreted by nearly every type of cell in the body, EVs have been implicated both in normal physiological processes, such as inflammation and cell proliferation, as well as the pathogenesis of various disease $\operatorname{states}^{[3,4]}$.

Within the cardiovascular niche, all cardiac cell types secrete and internalize EVs, suggesting they play an important role in intracardiac signaling. EVs stimulate angiogenesis, prevent apoptosis of cardiomyocytes, and exert anti-fibrotic effects following ischemic damage ${ }^{[1]}$. EVs may also partially account for the cardiovascular benefits of exercise, with one study showing a sharp increase in serum EV concentration in both humans and mice undergoing exercise stress testing ${ }^{[5]}$. This finding carries an important clinical implication: cells need not be in close proximity to communicate through EV cargo. Rather, systemic release

The Author(s) 2022. Open Access This article is licensed under a Creative Commons Attribution 4.0 International License (https://creativecommons.org/licenses/by/4.0/), which permits unrestricted use, sharing, adaptation, distribution and reproduction in any medium or format, for any purpose, even commercially, as long as you give appropriate credit to the original author(s) and the source, provide a link to the Creative Commons license, and indicate if changes were made. 
of circulating EVs can regulate cells and tissues distal from the initial site of secretion. Compared to traditional chemokines, EVs seem to be particularly suited to this type of signaling, as their membrane protects their cargo from rapid degradation and clearance, and proteins on the EV surface allow for trafficking to specific target cell types ${ }^{[6]}$. In contrast to the numerous stem cell-based therapies under investigation, intramyocardial (local) delivery of EVs does not seem to be necessary in order to elicit the desired response, which makes EV therapy a more attractive proposition for eventual clinical translation.

The influence of EVs may also help to explain the variability in results when stem cells are delivered to the failing heart. The heart is largely considered to be a post-mitotic organ, with an annual turnover of cardiomyocytes in the adult heart of less than $1 \%{ }^{[7]}$. This meager turnover is inadequate for repairing the damage following a myocardial infarction (MI) or other major injuries. Because of this, the "Holy grail" of stem cell therapy in the cardiac field for many years has been a replacement of lost contractile mass by the delivery of stem cells capable of differentiating into new cardiomyocytes in situ. Many cell populations have been suggested, with two of the most scrutinized candidates being c-kit+ cardiac progenitor cells (CPCs) and mesenchymal stem cells (MSCs). In recent years, lineage tracing experiments have firmly debunked the thought that CPCs may be cardiomyogenic, and while MSCs may have some capacity to transdifferentiate into cardiomyocytes given the appropriate cues, evidence of this differentiation upon delivery has been weak $^{[8,9]}$. In spite of this, numerous small animal studies have shown significant benefits to MSC and CPC delivery to the myocardium following MI, even when retention and engraftment of these cells are low, and most of the transplanted cells are washed out within a few days. This is reflected in the recently published results of the Combination of Mesenchymal and c-kit+ Cardiac Stem Cells As Regenerative Therapy for Heart Failure (CONCERT-HF) clinical trial, in which heart failure patients were dosed with either MSCs, CPCs, or the two cell types in combination via transendocardial injection ${ }^{[10]}$. Delivery of CPCs, either alone or in combination with MSCs, greatly reduced the incidence of major adverse coronary events, while MSCs, either alone or in combination with CPCs, showed significant quality of life improvements for patients.

These data beg the question: if MSCs and CPCs do not differentiate, and in many cases, are transient within the heart and quickly washed out, where do these benefits arise from? A growing body of evidence would suggest that paracrine factors, specifically the release of EVs by these stem cells, are responsible for the bulk of their pro-reparative effects. If this is the case, then delivery of isolated and concentrated EVs makes a great deal of sense compared to the delivery of cells. As previously hinted at, EVs can be delivered systemically by intravenous injection, obviating the need for delivery to the endocardium or myocardium. Additionally, immunogenicity of stem cells, particularly if derived from allogeneic sources, is a major cause of concern. One study on the immunogenicity of EVs in mice showed minimal toxicity or changes in immune markers in response to repeated dosing with EVs intravenously ${ }^{[1]}$. The relative ease of isolating, concentrating, and delivering EVs compared to stem cells, combined with a more promising safety profile, could see the field shift more towards EV-based therapies in the coming years.

Despite the promise shown by EVs in early in vitro studies, several obstacles remain for transition to clinical applications. Typically, EVs are isolated from ordinary monolayer cultured cells by ultracentrifugation of conditioned media to concentrate the EVs. The number of cells required to produce a clinically relevant dose of EVs and the labor involved in maintaining such a large quantity of cells make this process difficult to scale up ${ }^{[12]}$. Additionally, the use of fetal bovine serum (FBS) supplemented media in the production of EVs is unlikely to pass Food and Drug Administration clearance due to restrictions on the use of animal products in human therapeutics. 
A new study by Bellio et al. ${ }^{[13]}$ elegantly addresses both of these challenges. Utilizing a Quantum bioreactor manufactured by Terumo Blood and Cell Technologies, they were able to demonstrate a large-scale automated culture of MSCs. Consisting of a bundle of hollow fibers, the Quantum bioreactor is equal in surface area to 120 standard T-175 cell culture flasks. Starting with $30 \times 10^{6}$ MSCs, peak expansion was achieved within only 6-7 days. Once peak expansion was obtained, a small volume of conditioned media was withdrawn from the bioreactor every $24 \mathrm{~h}$ for concentration and isolation of EVs. Although the appropriate dose of EVs in humans has not been established, Bellio et al. ${ }^{[13]}$ postulate that based on large animal studies, the total quantity of EVs isolated from the bioreactor after $120 \mathrm{~h}$ would constitute 3-10 clinically relevant doses. Despite orders of magnitude higher throughput than traditional monolayer cell culture, this bioreactor is a small, commercially available benchtop unit that could be easily incorporated into a clinical laboratory.

In addition to demonstrating the abilities of the Quantum bioreactor for large-scale harvesting of EVs from MSCs, Bellio et al. ${ }^{[13]}$ also investigated the use of human platelet lysate (PLT) as a substitute for FBS supplementation in culture media. Comparison of EVs produced from MSCs grown in FBS supplemented media with those produced from MSCs grown in platelet lysate supplemented media suggest that both are appropriate, though with a few noteworthy differences. MSCs in PLT supplemented media actually reached peak expansion quicker than those grown in FBS. Additionally, there were no significant differences in size, quantity, or expression of surface markers CD63 and CD81 in the extracellular vesicles collected. Examination of the EV cargo showed approximately $90 \%$ of the identified miRs in common between the two groups, although 12 miRs in the PLT group and 15 miRs in the FBS group were identified as unique. As miRs primarily exert their effects through post-transcriptional silencing of mRNAs, bioinformatic analysis techniques can be used to identify unique mRNA targets for each miR sequence, which can then be examined for associations with known signaling pathways or specific outcomes such as fibrosis or angiogenesis. Using this analysis, 993 common mRNA targets were identified between the two groups, while 28 unique targets were identified in the PLT group, and 52 unique targets were identified in the FBS group. While the study points out that the copy numbers were relatively low for the unique miRs compared to the common miRs, suggesting that the mechanism of action of EVs in the two groups is largely shared, further investigation may be warranted to see whether the supplementation with either FBS or PLT alters miR cargo.

Finally, Bellio et al ${ }^{[13]}$ went on to evaluate the efficacy of their isolated EVs in a murine MI model. Mice were subjected to left coronary artery occlusion, followed by weekly intra-jugular injections of EVs. Cardiac function was periodically measured by echocardiography, and after 8 weeks, the hearts were explanted and stained to evaluate infarct size and fibrosis. After 8 weeks, EVs isolated from both FBS and PLT culture showed improved cardiac function as measured by ejection fraction and stroke volume. Interestingly, the results for reduction in infarct size were more mixed, with PLT-EVs showing a decrease in infarct size over the placebo group, but FBS-EVs showing no difference in infarct size. Bollio et al ${ }^{[13]}$ speculate this may be due to decreased retention of FBS-EVs in the myocardium, but do not speculate further as to the specific mechanism involved. This difference in infarct size, therefore, may warrant further investigation.

This study by Bellio et al. ${ }^{[13]}$ represents a major step towards scaling and adapting the manufacture and concentration of EVs to the clinic and, most importantly, paves the way for future clinical trials, which are sure to provide an additional wealth of data. However, a few open questions remain which may be worthy of further investigation. In particular, while the composition of miR cargoes was studied in detail, this tells only a part of the story of the biological activity of EVs. While exosomes likely exert most of their function through post-transcriptional regulation of mRNA, vis-à-vis the delivery of miRs, the protein and lipid 
composition of the EV membrane also seems to play an important role, particularly in the targeting of miRs to particular cell types. Some groups have even leveraged this to their advantage, and engineering targeted exosomes by manipulating the surface protein ${ }^{[14]}$. In terms of this study, the difference in infarct size between PLT-EVs and FBS-EVs may be explained by differences in surface protein composition, rather than differences in miR cargo. Proteomic and lipidomic analysis, along with uptake studies on cardiomyocytes, endothelial cells, and cardiac fibroblasts, may help to further elucidate the cause of this difference. In any case, the finding that PLT-EVs are more beneficial than FBS-EVs is good news for clinical translation.

A final consideration that may be worthy of future evaluation is adapting the bioreactor culture to mimic hypoxia. A number of studies have found that hypoxia drives changes in exosome release from MSCs, specifically the enrichment of miRs that elicit an anti-apoptotic effect ${ }^{[15,16]}$. This may be an endogenous survival mechanism that serves to protect cardiomyocytes during periods of ischemia. As such, hypoxia challenging MSCs has become a commonplace strategy for boosting their efficacy when harvesting EVs. It is unclear whether this particular bioreactor is capable of hypoxic culture. However, if it is possible to combine bioreactor culture of MSCs with hypoxia, it would be interesting to evaluate whether hypoxic EVs may elicit even more reparative benefits.

In summation, Bellio et al. ${ }^{[13]}$ have demonstrated a powerful methodology for the scaling up of MSC-EV manufacture for the treatment of MI and heart failure. Though relatively few phase I clinical trials have evaluated the use of MSC-EVs in various disease states, to date, MSC-EVs have not been evaluated in humans for the treatment of MI or heart failure. The advances in bioreactor culture and elimination of FBS from the culture with no deleterious effects of note will surely advance the field closer to clinical trials of MSC-EVs in the heart.

\section{DECLARATIONS}

\section{Authors' contributions}

Drafted and prepared the manuscript: Robeson MJ, Davis ME

\section{Availability of data and materials}

Not applicable.

\section{Financial support and sponsorship}

None.

\section{Conflicts of interest}

Both authors declared that there are no conflicts of interest.

\section{Ethical approval and consent to participate}

Not applicable.

\section{Consent for publication}

Not applicable.

\section{Copyright}

(c) The Author(s) 2022. 


\section{REFERENCES}

1. Chistiakov DA, Orekhov AN, Bobryshev YV. Cardiac extracellular vesicles in normal and infarcted heart. Int J Mol Sci 2016;17:63. DOI PubMed PMC

2. Rashed MH, Bayraktar E, Helal GK, et al. Exosomes: from garbage bins to promising therapeutic targets. Int J Mol Sci 2017;18:538. DOI PubMed PMC

3. Zhang Y, Hu YW, Zheng L, Wang Q. Characteristics and roles of exosomes in cardiovascular disease. DNA Cell Biol 2017;36:202-11. DOI PubMed

4. Zhang L, Yu D. Exosomes in cancer development, metastasis, and immunity. Biochim Biophys Acta Rev Cancer 2019;1871:455-68. DOI PubMed PMC

5. Bei Y, Xu T, Lv D, et al. Exercise-induced circulating extracellular vesicles protect against cardiac ischemia-reperfusion injury. Basic Res Cardiol 2017;112:38. DOI PubMed PMC

6. Vogt S, Stadlmayr G, Grillari J, Rüker F, Wozniak-knopp G. Engineering of surface proteins in extracellular vesicles for tissuespecific targeting. In: Shiomi N, editor. Current topics in biochemical engineering. IntechOpen; 2019. DOI

7. Bergmann O, Bhardwaj RD, Bernard S, et al. Evidence for cardiomyocyte renewal in humans. Science 2009;324:98-102. DOI PubMed PMC

8. Hatzistergos KE, Quevedo H, Oskouei BN, et al. Bone marrow mesenchymal stem cells stimulate cardiac stem cell proliferation and differentiation. Circ Res 2010;107:913-22. DOI PubMed PMC

9. Molkentin JD. Letter by Molkentin regarding article, "The absence of evidence is not evidence of absence: the pitfalls of Cre KnockIns in the c-Kit Locus". Circ Res 2014;115:e21-3. DOI PubMed PMC

10. Bolli R, Mitrani RD, Hare JM, et al; Cardiovascular Cell Therapy Research Network (CCTRN). A Phase II study of autologous mesenchymal stromal cells and c-kit positive cardiac cells, alone or in combination, in patients with ischaemic heart failure: the CCTRN CONCERT-HF trial. Eur J Heart Fail 2021;23:661-74. DOI PubMed PMC

11. Zhu X, Badawi M, Pomeroy S, et al. Comprehensive toxicity and immunogenicity studies reveal minimal effects in mice following sustained dosing of extracellular vesicles derived from HEK293T cells. J Extracell Vesicles 2017;6:1324730. DOI PubMed PMC

12. Colao IL, Corteling R, Bracewell D, Wall I. Manufacturing exosomes: a promising therapeutic platform. Trends Mol Med 2018;24:242-56. DOI PubMed

13. Bellio MA, Kanashiro-takeuchi RM, Takeuchi L, et al. Systemic delivery of large-scale manufactured Wharton's Jelly mesenchymal stem cell-derived extracellular vesicles improves cardiac function after myocardial infarction. J Cardiovasc Aging 2022. DOI

14. Liang Y, Xu X, Li X, et al. Chondrocyte-targeted microRNA delivery by engineered exosomes toward a cell-free osteoarthritis therapy. ACS Appl Mater Interfaces 2020;12:36938-47. DOI PubMed

15. Cheng H, Chang S, Xu R, et al. Hypoxia-challenged MSC-derived exosomes deliver miR-210 to attenuate post-infarction cardiac apoptosis. Stem Cell Res Ther 2020;11:224. DOI PubMed PMC

16. Chen J, Chen J, Cheng Y, et al. Mesenchymal stem cell-derived exosomes protect beta cells against hypoxia-induced apoptosis via miR-21 by alleviating ER stress and inhibiting p38 MAPK phosphorylation. Stem Cell Res Ther 2020;11:97. DOI PubMed PMC 